\title{
Differential gene expression profiling of esophageal adenocarcinoma
}

\author{
Zane T. Hammoud, MD, ${ }^{\text {a }}$ Sunil Badve, MD,${ }^{\mathrm{b}, \mathrm{c}, \mathrm{d}}$ Qianqian Zhao, MS, ${ }^{\mathrm{e}}$ Lang Li, PhD, ${ }^{\mathrm{e}}$ Romil Saxena, MD, \\ Mangesh A. Thorat, MD, ${ }^{\mathrm{b}}$ Akira Morimiya, BS, ${ }^{\mathrm{b}}$ Karen M. Rieger, MD, ${ }^{\mathrm{a}}$ and Kenneth A. Kesler, MD ${ }^{\mathrm{a}}$
}

\begin{abstract}
Background: Differential gene expression offers an attractive means by which to study genes that may be involved in disease development and/or progression. We performed quantitative gene expression in various stages of esophageal adenocarcinoma, treated exclusively by surgery with complete 2-field lymphadenectomy, in an attempt to discern genes involved in disease progression as well as genes that may predict survival.
\end{abstract}

\begin{abstract}
Methods: Gene expression profiling was accomplished by cDNA-mediated annealing, selection, extension, and ligation (DASL) assay. RNA was extracted from 89 archived formalin-fixed, paraffin-embedded esophageal adenocarcinoma tissues. DASL assay was performed with the Sentrix Universal Array (Illumina Corp, San Diego, Calif) of 502 known cancer-related genes. Bioinformatics tools were used to determine significant differential gene expression in T1-2 versus T3-4 tumors and tumors without lymph node involvement (N0) versus tumors with lymph node involvement $(\mathrm{N}+)$. Gene expression was also correlated with overall survival.

Results: Twenty-one genes were overexpressed in T1-2 compared with T3-4 tumors (false discovery rate of 0). Underexpression of 1 gene was seen in $\mathrm{N}+$ compared with N0 tumors (false discovery rate of 0 ). For overall survival, underexpression of 9 genes correlated with long survival.

Conclusions: Using differential gene expression of 502 known cancer genes, we identified genes that may be involved at various stages in the progression of esophageal adenocarcinoma. We also identified genes that may correlate with prolonged survival and, thus, may serve as prognostic markers. These findings may provide further insight into the mechanisms of development and/or progression of esophageal adenocarcinoma. Prospective studies are needed to verify the prognostic value of these genes.
\end{abstract}

The incidence of esophageal adenocarcinoma has increased markedly over the past 30 years. In the Western world, adenocarcinoma is now more common than esophageal squamous cell carcinoma. ${ }^{1}$ Surgical resection, with or without the addition of chemotherapy and/or radiation therapy, remains the cornerstone of therapy for esophageal adenocarcinoma and represents the best curative treatment option. However, despite improvements in preoperative staging and in operative morbidity and mortality, the prognosis of patients with adenocarcinoma remains relatively poor. A variety of factors have been explored to determine the biologic behavior of esophageal adenocarcinoma. Widely known prognostic factors, such as stage of tumor and lymph node involvement, are included in the TNM staging system. In addition, other factors such as the number of positive lymph nodes, grade of differentiation, and response to neoadjuvant chemoradiation have been shown to have additional prognostic value., ${ }^{2,3}$ The description of molecular and/or genetic changes involved in carcinogenesis has led to opportunities to explore the impact of such changes on

\footnotetext{
From the Departments of Surgery, Thoracic Surgery Division, ${ }^{a}$ Pathology and Laboratory Medicine, ${ }^{\mathrm{b}}$ Internal Medicine, ${ }^{\mathrm{c}}$ Translational Genomics Core of Indiana University Cancer Center, ${ }^{\mathrm{d}}$ and Bioinformatics, ${ }^{\mathrm{e}}$ Indiana University School of Medicine, Indianapolis, Ind.

Received for publication June 2, 2008; revisions received July 24, 2008; accepted for publication Aug 31, 2008.

Address for reprints: Zane T. Hammoud, MD, Department of Surgery, Thoracic Surgery Division, Indiana University School of Medicine, 545 Barnhill Dr, EH 215, Indianapolis, IN 46202 (E-mail: zhammoud@iupui.edu).

J Thorac Cardiovasc Surg 2009;137:829-34

$0022-5223 / \$ 36.00$

Copyright (C) 2009 by The American Association for Thoracic Surgery

doi:10.1016/j.jtcvs.2008.08.052
}

clinical behavior. A better understanding of these changes may identify additional factors that have the potential to improve prognostication and to influence additional therapy.

Carcinogenesis is a complex process that involves multiple genetic alterations. Many methods have been developed to study these changes and to delineate their potential impact on clinical behavior. The sequentiation of the human genome, combined with high throughput technologies, has led to the ability to describe these genetic alterations in a quantitative manner, thus allowing the development of tumor "profiles" that can distinguish subsets of disease, predict response to therapy, and possibly outcome. In esophageal adenocarcinoma, gene expression profiling using DNA microarrays has been used to compare adenocarcinoma with squamous cell carcinoma and with Barrett esophagus, establishing the presence of unique gene expression profiles capable of discriminating between these diseases. ${ }^{4}$

We performed gene expression profiling on formalinfixed, paraffin-embedded (FFPE) esophageal adenocarcinomas using the cDNA-mediated annealing, selection, extension and ligation (DASL) assay. Quantitative gene expression was performed in various stages of adenocarcinoma, treated exclusively by surgery with complete 2-field lymphadenectomy, in an attempt to discern genes involved in disease progression as well as genes that may predict survival.

\section{PATIENTS AND METHODS \\ Patients and Samples}

The study was approved by the Indiana University Institutional Review Board. From 1990 to 2005, 138 patients with the diagnosis of esophageal 


\section{Abbreviations and Acronyms \\ $\mathrm{DASL}=\mathrm{cDNA}$-mediated annealing, selection, extension, and ligation \\ DNA $=$ deoxyribonucleic acid \\ FFPE $=$ formalin-fixed, paraffin-embedded \\ RNA $=$ ribonucleic acid}

TABLE 1. Pathologic TNM stage of 89 specimens used for analysis

\begin{tabular}{lr}
\hline TNM stage & No. \\
\hline T1 N0 & 10 \\
T1 N1 & 5 \\
T2 N0 & 4 \\
T2 N1 & 7 \\
T3 N0 & 9 \\
T3 N1 & 51 \\
T4 N1 & 2 \\
Tx N1 & 1 \\
\hline
\end{tabular}

adenocarcinoma, treated exclusively by surgery with complete 2 -field lymphadenectomy as initial therapy at the Indiana University Medical Center, were identified from a prospectively maintained database. Representative hematoxylin and eosin-stained slides from all patients were reviewed by two pathologists (S.B. and R.S.). A section with a minimum of $1 \mathrm{~cm}^{2}$ tumor-bearing area with at least $70 \%$ tumor was the selection criterion for inclusion in the study. Forty-one specimens did not fulfill this criterion and were excluded. Specimens with the minimum $1 \mathrm{~cm}^{2}$ tumor-bearing area but with adjacent or surrounding stromal/granulation tissue were included; these specimens were subjected to manual macrodissection with hematoxylin and eosin slides used to facilitate macrodissection of the tumor-bearing area. The corresponding paraffin blocks were obtained from the Indiana University Department of Pathology. Three $10-\mu \mathrm{m}$ sections were obtained from each selected paraffin block (one block per case) on noncharged glass slides taking due precautions to avoid nucleic acid contamination. Sections were deparaffinized with CitriSolv cleaning agent (Fisher Scientific Company, Fair Lawn, NJ) and scraped off from the slide into a microcentrifuge tube. For sections requiring macrodissection, only the tumor-bearing areas were scraped off using visual matching with marked hematoxylin and eosin slides. RNA was extracted from a total of 97 cases using High Pure RNA Paraffin Kit (Roche Applied Bioscience, Indianapolis, Ind). Eighty-nine cases fulfilled the RNA requirement of $200 \mathrm{ng} / 5 \mu \mathrm{L}$ for the assay and served as the basis for the study. RNA was pre-qualified using iScript (Bio-Rad Laboratories Inc, Hercules, Calif) to reverse transcribe and SYBR Green Master Mix (Applied Biosystems, Foster City, Calif) to perform quantitative polymerase chain reaction for RPL13a gene. DASL assay was performed with the Sentrix Universal Array (Illumina Corp., San Diego, Calif) of 502 known cancer genes as per the manufacturer's instructions. ${ }^{5}$ Technical duplicates for four samples were also included in the assay.

Clinical follow-up was obtained from office visits and through telephone contact. The pathologic TNM stage, date of surgery, date of death where applicable, and the date of last follow-up were recorded. Specimens were grouped into $\mathrm{T} 1$ and $\mathrm{T} 2$ tumors (T1-2) or $\mathrm{T} 3$ and $\mathrm{T} 4$ tumors (T3-4) to delineate genes that may be involved in advancing $\mathrm{T}$ stage as well as into tumors without lymph node involvement (N0) or with lymph node involvement $(\mathrm{N}+)$ to delineate genes that may be involved in lymph node metastasis. On the basis of survival from the date of surgery, Kaplan-Meier survival curves were calculated. A comparison was made on the basis of patient overall survival to delineate genes that may confer a survival advantage.

\section{Statistical Analysis}

Gene expression data were normalized at the median level. Hierarchical clustering and singular value decomposition methods were applied to detect the outliers for quality control purposes. The gene expression data was then correlated with T stage (1-2 vs 3-4) and with lymph node status (negative vs positive) through significant analysis of microarray. ${ }^{6} \mathrm{~T}$ stage, lymph node status, and gene expression were correlated with patient overall survival through log-rank tests. Patients who died perioperatively as well as patients who died of noncancer causes were censored in the overall survival analysis. The false discovery rate, ${ }^{7}$ an estimate of the proportion of errors committed by falsely rejecting null hypotheses and widely used in genome wide correlative studies, was calculated for each gene. Top-ranked genes were selected by a prespecified false discovery rate (q value) of 0.20 . Gene set analysis

Table 1 lists the TNM pathologic stage of all 89 specimens analyzed. There were 26 T1-2 and 62 T3-4 specimens whereas there were $23 \mathrm{~N} 0$ and $66 \mathrm{~N}+$ specimens. The median follow-up was 25 months (range 2-132 months) and 4 patients were lost to follow-up. The median survival for the entire group was 2.18 years (Figure 1). Analysis of T1-2 specimens compared with T3-4 specimens demonstrated up-regulation (overexpression) of a total of 63 genes, 21 of which had a false discovery rate (q value) of 0 . Tables $2 \mathrm{~A}$ and $2 \mathrm{~B}$ demonstrate the results of this analysis and the genes identified. Table 3 demonstrates results of the analysis of $\mathrm{N} 0$ specimens compared with $\mathrm{N}+$ specimens. A total of 16 genes were overexpressed and 1 gene $(M Y B)$ was underexpressed (down-regulated); only the underexpressed gene had a false discovery rate of 0 . For the survival analysis, no overexpressed genes correlated with prolonged survival. However, underexpression of 9 genes correlated with prolonged survival (Table 4). Figure 2 demonstrates a typical survival curve for one of these genes (CSPG2 or chondroitin sulfate proteoglycan core protein 2); the other 8 genes demonstrated similar survival curves. Figures 3 and 4

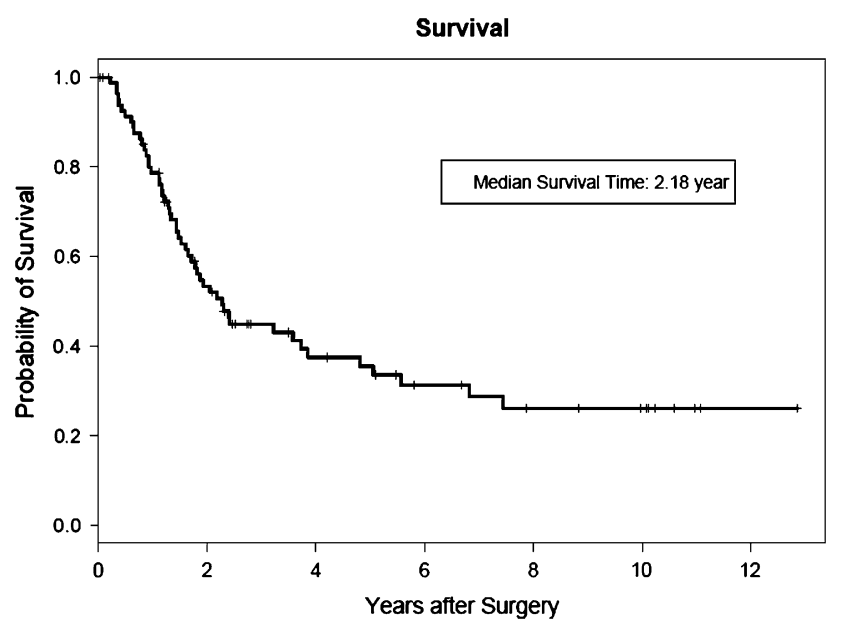

FIGURE 1. Kaplan-Meier survival curve of all 89 patients. was performed among the top ranked genes. ${ }^{8}$

\section{RESULTS}


TABLE 2A. Analysis of T1-2 tumors compared with T3-4 tumors

\begin{tabular}{|c|c|c|}
\hline Gene name & Fold change & q Value $(\%)$ \\
\hline FGF6 & 1.21921 & 1.91959 \\
\hline TSCl & 1.20543 & 1.91959 \\
\hline$W N T 8 B$ & 1.12815 & 1.91959 \\
\hline$C D C 25 C$ & 1.18296 & 1.91959 \\
\hline$M C F 2$ & 1.20778 & 1.91959 \\
\hline$D C C$ & 1.19554 & 1.91959 \\
\hline$M O S$ & 1.19179 & 1.91959 \\
\hline IL13 & 1.14319 & 1.91959 \\
\hline$F G F 3$ & 1.33406 & 1.91959 \\
\hline$R A D 54 B$ & 1.13327 & 1.91959 \\
\hline$N R A S$ & 1.10673 & 1.91959 \\
\hline$G 22 P 1$ & 1.17275 & 1.91959 \\
\hline$T D G F 1$ & 1.19453 & 2.63944 \\
\hline$I G F 1 R$ & 1.16352 & 2.63944 \\
\hline$I F N G$ & 1.15091 & 2.63944 \\
\hline$T P 73$ & 1.20165 & 3.24854 \\
\hline$I G F B P I$ & 1.13768 & 3.24854 \\
\hline$P G R$ & 1.11939 & 3.24854 \\
\hline EPHAl & 1.1288 & 4.41953 \\
\hline$T G F B 1$ & 1.14399 & 4.41953 \\
\hline CSF3 & 1.17331 & 4.41953 \\
\hline$B L M$ & 1.1637 & 4.41953 \\
\hline$G M L$ & 1.19125 & 5.03894 \\
\hline MYCLI & 1.13767 & 5.63081 \\
\hline ERCCl & 1.15039 & 5.63081 \\
\hline CUL2 & 1.09062 & 5.63081 \\
\hline$E R C C 2$ & 1.13815 & 7.22374 \\
\hline TYRO3 & 1.14349 & 7.22374 \\
\hline NOTCH4 & 1.07357 & 7.22374 \\
\hline$R R A S$ & 1.14274 & 7.22374 \\
\hline NOTCH2 & 1.09151 & 7.22374 \\
\hline FER & 1.12716 & 7.22374 \\
\hline$D D B 2$ & 1.10884 & 7.22374 \\
\hline MTHFR & 1.09651 & 7.22374 \\
\hline$C O L 4 A 3$ & 1.10613 & 7.22374 \\
\hline$W N T 1$ & 1.08621 & 7.22374 \\
\hline$C D C 25 A$ & 1.09301 & 7.22374 \\
\hline TRAF3 & 1.09166 & 9.04952 \\
\hline$W T 1$ & 1.14302 & 9.04952 \\
\hline$R E T$ & 1.07779 & 9.04952 \\
\hline ERCC6 & 1.13908 & 9.04952 \\
\hline FLT3 & 1.1193 & 9.04952 \\
\hline
\end{tabular}

Overexpression of 42 genes with q value greater than 0 .

demonstrate the survival curves of patients with T1-2 versus T3-4 tumors and of patients with $\mathrm{N} 0$ versus $\mathrm{N}+$ tumors, respectively.

\section{DISCUSSION}

Gene expression profiling has become a powerful tool for the discovery of unique patterns that can distinguish a variety of disease states. In combination with statistical analysis, DNA microarrays have been used to study various cancers and to categorize them on a molecular level. The microar-
TABLE 2B. Results of T1-2 tumors compared with T3-4 tumors

\begin{tabular}{lc}
\hline Gene name & Fold change \\
\hline MYCN & 1.31278 \\
$F G F 8$ & 1.20703 \\
$C Y P 1 A 1$ & 1.23692 \\
$E R B B 4$ & 1.30322 \\
LTA & 1.18221 \\
$A B C G 2$ & 1.25264 \\
ERTV1 & 1.22501 \\
TNFSF6 & 1.17038 \\
NTRK2 & 1.1634 \\
FGF5 & 1.18971 \\
$C H E K 1$ & 1.23283 \\
NAT2 & 1.17645 \\
PMS1 & 1.18019 \\
MAS1 & 1.18816 \\
SIAH1 & 1.12057 \\
$P L G$ & 1.19926 \\
FANCA & 1.20802 \\
$D K C 1$ & 1.16013 \\
ALK & 1.23865 \\
MPL & 1.19515 \\
RARB & 1.22884 \\
\hline Overexpression of 21 genes demonstrated a false discovery rate (q value) of 0.
\end{tabular}

rays have been used to generate expression profiles that may predict disease prognosis, for example, breast cancer, as well as to delineate tumor pathway profiles. ${ }^{9}$ In esophageal cancer, gene expression profiling has been used to differentiate normal esophagus, Barrett esophagus, and adenocarcinoma. ${ }^{4}$ Such reports have focused mainly on comparing adenocarcinoma with other tissues in an attempt to

TABLE 3. Analysis of $\mathbf{N}_{0}$ tumors compared with $\mathbf{N}_{+}$tumors

\begin{tabular}{llr}
\hline Gene name & Fold change & q value (\%) \\
\hline Overexpressed & & \\
SERPINE1 & 1.232970722 & 41.30536677 \\
PTHLH & 1.121515751 & 41.30536677 \\
ETV1 & 1.163101391 & 41.30536677 \\
WRN & 1.119533194 & 41.30536677 \\
ABCC2 & 1.180818778 & 41.30536677 \\
ERG & 1.149993726 & 41.30536677 \\
MPL & 1.147600832 & 41.30536677 \\
PTCH2 & 1.174312145 & 41.30536677 \\
ERBB4 & 1.185113229 & 41.30536677 \\
ARHH & 1.098985548 & 41.30536677 \\
WNT10B & 1.165125114 & 41.30536677 \\
VAV1 & 1.08979885 & 41.30536677 \\
ICAM1 & 1.121376886 & 41.30536677 \\
TNFSF6 & 1.117215827 & 41.30536677 \\
IL12A & 1.097115543 & 41.30536677 \\
CSF3 & 1.160386817 & 41.30536677 \\
Underexpressed & & 0 \\
MYB & 0.749712525 & \\
\hline A &
\end{tabular}

A total of 16 genes were overexpressed and 1 gene was underexpressed. 
TABLE 4. Underexpressed genes that correlated with prolonged survival

\begin{tabular}{lcc}
\hline Gene name & q Value $(\%)$ & $\boldsymbol{P}$ value \\
\hline CSPG2 & 7.1 & .0001 \\
COL1A1 & 9.1 & .0004 \\
IGFBP3 & 7.1 & .0004 \\
SPP1 & 9.5 & .0007 \\
$M M P 14$ & 8.3 & .0008 \\
CDH11 & 15.7 & .0002 \\
TIMP1 & 14.1 & .0002 \\
TGFB3 & 18.8 & .0003 \\
MYB & 19.7 & .0004 \\
\hline
\end{tabular}

identify gene profiles that can distinguish, that is, "diagnose," adenocarcinoma from other tissue types. Dahlberg and associates ${ }^{10}$ used microarray analysis of 12,000 genes and noted that only a small subset of genes distinguished adenocarcinoma from normal controls. In this report, the authors compared 10 esophageal adenocarcinoma samples with 7 normal mucosa samples and found that 64 genes (of a total of 12,000) were potentially involved in the transition from normal epithelium to adenocarcinoma. Although the genes identified in this report did not correlate with our results, it is important to note that our study compared tumors with other tumors as opposed to normal tissues and that different genes may be involved in the transition from normal to tumor than from tumor to more invasive tumor. Furthermore, the number of specimens analyzed was relatively small whereas the number of genes analyzed was very large; thus, statistical analysis of such data may be difficult to compare with our data. Selaru and colleagues ${ }^{11}$ also used cDNA microarrays and reported that various gene clusters were able to distinguish Barrett, adenocarcinoma, and squamous cell carcinoma. This report used a small number of samples and generated gene "clusters" that correlated

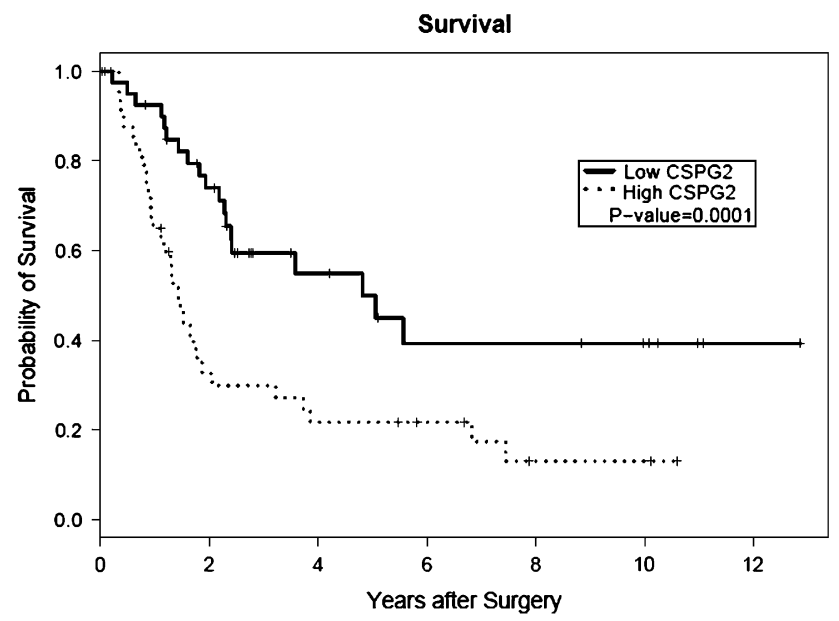

FIGURE 2. Kaplan-Meier survival curve of patients based on expression of $C S P G 2$.

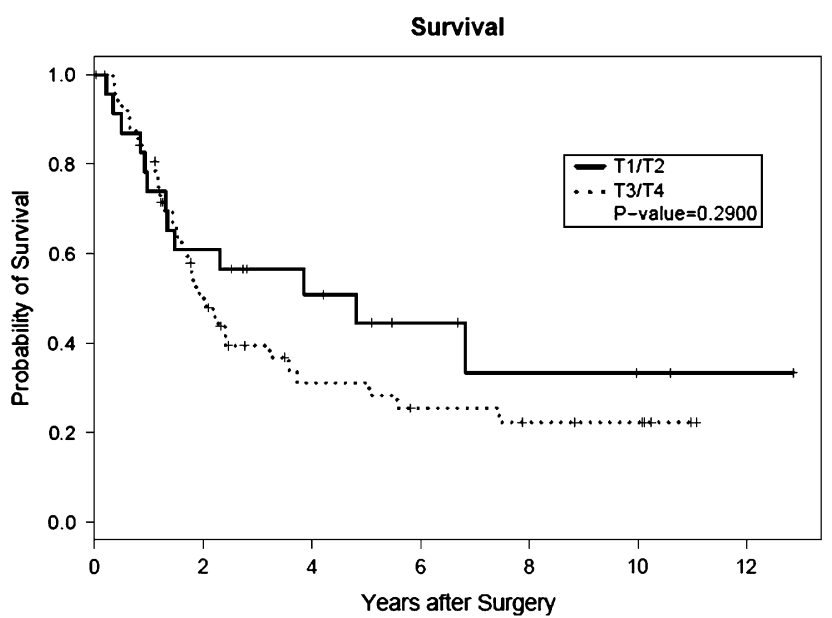

FIGURE 3. Kaplan-Meier survival curve of patients based on $\mathrm{T}$ stage.

with tissue histologic characteristics. The authors did not identify specific genes. Greenawalt, ${ }^{4} \mathrm{Hao},{ }^{12}$ and their associates used genome-wide gene expression profiling to identify candidate genes that may play a role in esophageal cancer carcinogenesis. These reports, unlike ours, again focused on a comparative analysis between Barrett and adenocarcinoma. In another report, Kimchi and colleagues ${ }^{13}$ reported gene expression profiling of Barrett and adenocarcinoma and found that 21 genes may represent potential markers of disease progression; six of these genes showed significant correlation with progression from normal to Barrett to adenocarcinoma. Common to all of these studies is the underlying assumption that Barrett esophagus is the genetic precursor to invasive adenocarcinoma. Guo and coworkers ${ }^{14}$ reported that distinctive microRNA expression profiles may relate to patient survival in esophageal squamous cell carcinoma, whereas Feber and colleagues ${ }^{15}$ reported microRNA expression profiles that distinguished normal tissue from

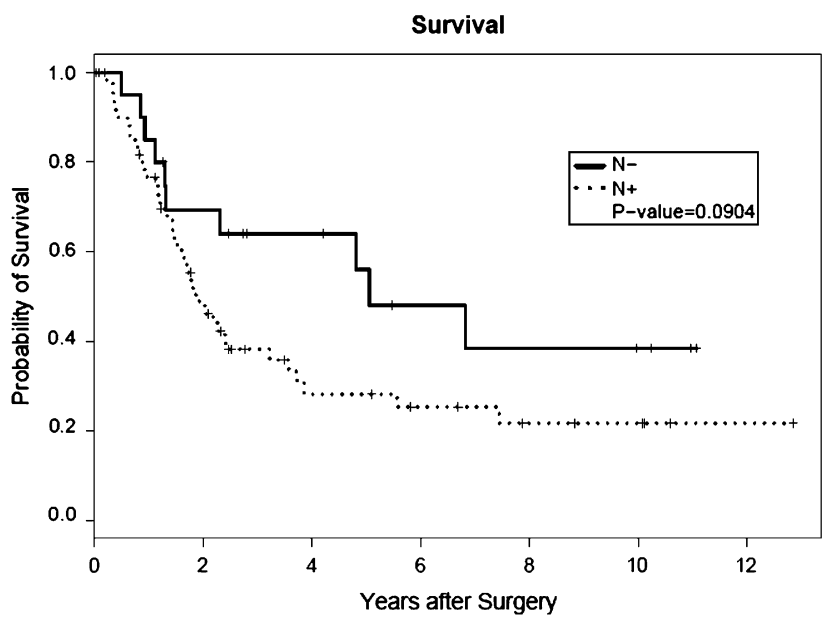

FIGURE 4. Kaplan-Meier survival curve of patients based on lymph node involvement. 
esophageal adenocarcinoma. Luthra and associates ${ }^{16}$ reported an association with pathologic response to neoadjuvant chemoradiation and unique gene expression profiles. All of these reports have demonstrated the potential utility of gene expression profiling in esophageal adenocarcinoma. However, none has attempted to correlate gene expression with survival in adenocarcinoma.

We performed gene expression profiling using the DASL assay of 502 genes that are known to be involved in carcinogenesis. Unlike the widely reported DNA microarrays, this assay identifies differences in gene expression of genes whose pathways and roles in carcinogenesis are relatively well defined. The other major advantage of this technology is the ability to perform analysis on archived FFPE specimens. DNA microarrays, although useful, typically require a large amount of fresh or frozen tissue for analysis. Although such patient tissue may be readily available at institutions that have made efforts to store the tissue, it is usually difficult to obtain meaningful long-term clinical data owing to the short duration of storage. In addition, some of this tissue is collected from patients who have undergone preoperative chemotherapy and/or radiation therapy, thus potentially altering the genetic profile of the cancer. However, there is a vast supply of FFPE tissue from patients whose clinical outcome is known due to the duration of storage of FFPE at most institutions. Tissue from biopsy specimens performed before any therapy and stored as FFPE also present the opportunity to study tumor characteristics without the potentially confounding variable of neoadjuvant therapy. The ability to perform gene expression profiling using FFPE greatly facilitates retrospective studies that can correlate gene expression with tumor histologic type as well as clinical outcome. This enables the analysis of specimens whose clinical outcome is known, that is, specimens from patients who have follow-up of several years' duration. In addition, analysis of normal tissue is unnecessary, inasmuch as all genes in the DASL array would be expected to be differentially expressed compared with normal tissue.

Combined with bioinformatics tools, we compared differential gene expression of pathologically staged $\mathrm{T} 1$ and $\mathrm{T} 2$ esophageal adenocarcinomas versus those staged as $\mathrm{T} 3$ and T4 in an attempt to identify genes that may correlate with depth of invasion. We identified a total of 63 genes that were overexpressed in the T3-4 tumors; 21 of these genes had a false discovery rate of 0 , indicating a high likelihood of significance in progression of depth of invasion. These genes include the N-myc proto-oncogene, a gene involved in apoptosis (LTA), a gene that belongs in the epidermal growth factor family (ERBB4), as well as other oncogenes. For the determination of genes that may be involved in lymph node involvement, our analysis showed that the underexpression of 1 gene, $M Y B$, had a false discovery rate of 0 , whereas the overexpression of 16 other genes had higher false discovery rates. The $M Y B$ gene has been reported to play a critical role in the differentiation/proliferation of hematopoeitic as well as other cell types and to have latent transforming activity. ${ }^{17}$ This gene has also been reported to be involved in progression of colon cancer. ${ }^{18}$ The gene expression profile of increasing $\mathrm{T}$ stage and that of $\mathrm{N}+$ were noted to have some common genes (ERBB4, ETV1, TNFSF6, MPL), suggesting that such genes have the potential to identify tumors of advancing stage. The gene expression profiles were correlated with overall survival and showed that underexpression of 9 genes conferred a survival advantage. Our analysis further indicated that gene expression was superior to $\mathrm{T}$ stage as well as lymph node involvement in predicting overall survival, for the survival curves of patients with T1-2 versus T3-4 tumors as well of patients with $\mathrm{N} 0$ versus $\mathrm{N}+$ tumors did not demonstrate a statistically significant difference in survival. Some of these genes have been correlated with survival in other types of cancers. The SPPI (osteopontin) gene has been reported to be a determinant of decreased survival in gastric cancer. ${ }^{19}$ The $C S P G 2$ gene is reported to be a target for TP53 while increased expression of IGFBP3 (insulin-like growth factor binding protein 3 ) has been reported to be involved in esophageal tumor growth. ${ }^{20,21}$ The heretofore mentioned $M Y B$ gene is also involved in tumor progression. Thus, underexpression of such genes makes intuitive sense in terms of conferring a survival advantage.

There are several limitations to our study. We did not use a strategy to have training and validation data sets. Although this would be highly desirable, doing so would have led to an even smaller number of samples in each category, thus raising questions about the statistical validity of our findings. We used an arbitrary cutoff of $70 \%$ tumor in the selection of our specimens. It is possible that the inclusion of normal tissue in our analysis may have led to inclusion of normal tissue gene expression. In theory, microdissection, for example, laser capture, may lead to the extraction of more pure tumor RNA and, perhaps, lead to a more accurate reflection of tumor gene expression. However, most of the specimens analyzed contained greater than $70 \%$ tumor tissue and, for those specimens that had close to the $70 \%$ cutoff, macrodissection was performed in an attempt to reduce the amount of normal tissue as much as possible. It may be more appropriate to perform comparison of $\mathrm{T} 1$ versus $\mathrm{T} 2, \mathrm{~T} 2$ versus $\mathrm{T} 3$, and so on, to obtain a more accurate reflection of the profile of increasing depth of invasion. However, we did not have sufficient numbers of tumors for each $\mathrm{T}$ state to perform a meaningful analysis. Inasmuch as the majority of tumors in our analysis were T3, we believed that comparison of tumors that have partially invaded the esophageal wall, that is, $\mathrm{T} 1$ and $\mathrm{T} 2$, with those that have fully penetrated the esophageal wall would be an accurate reflection of increasing depth of invasion. It is also possible that patients with an increasing number of involved lymph nodes have differential gene expression. Again, however, such an analysis was not 
performed owing to the small numbers involved. Our data did not reveal a statistically significant difference in survival with advancing $\mathrm{T}$ stage or with positive lymph node involvement. With regard to T stage, our combination of T1 and T2 tumors and of T3 and T4 tumors may obviously have influenced such a result. However, it is also now apparent that correlation of survival with $\mathrm{T}$ stage alone is imprecise, for within each $\mathrm{T}$ stage (particularly early $\mathrm{T}$ stage) subcategories exist that may better determine survival. It would be ideal to perform survival analysis based on $\mathrm{T} 1$ versus $\mathrm{T} 2$ versus $\mathrm{T} 3$ versus T4; however, the numbers involved are too small for a statistically valid analysis of this sort. Similarly, although the $\mathrm{N}+$ versus N0 survival analysis trended toward statistical significance, we did not observe a significant difference in survival in patients with $\mathrm{N}+$ versus $\mathrm{N} 0$ tumors. This observation may be due to the fact that $\mathrm{T}$ stage was not considered in this analysis. Furthermore, the number of lymph nodes involved was also not considered; this has been shown to influence outcomes as well.

In summary, we have performed gene expression profiling of esophageal adenocarcinomas using a technique that uses FFPE tissue and have shown correlation between a limited number of genes and increasing depth of invasion, lymph node involvement, and overall survival. The genes may be involved in esophageal adenocarcinoma carcinogenesis and may serve as potential markers of disease progression and/or prognosis. These genes may also be involved with other genes, not yet discovered, in pathways that are critical to carcinogenesis. Such genes may serve as potential targets for future therapy, as markers of disease recurrence, or as further aids in the refinement of esophageal cancer staging. Further validation of these data in a prospective manner is needed to determine the utility of these profiles and of the genes identified.

\section{References}

1. Devesa SS, Blo WJ, Fraumeni JF Jr. Changing patterns in the incidence of esophageal and gastric carcinoma in the United States. Cancer. 1998;83:2049-53.

2. Wijnhoven BPL, Tran KTC, Esterman A, Watson DI, Tilanus HW. An evaluation of prognostic factors and tumor staging of resected carcinoma of the esophagus. Ann Surg. 2007;245:717-25.

3. Rice TW, Blackstone EH, Rybicki LA, Adelstein DJ, Murthy SC, DeCamp MM, et al. Refining esophageal cancer staging. J Thorac Cardiovasc Surg. 2003;125: 1103-13.
4. Greenawalt DM, Duong C, Smyth GK, Ciaverella ML, Thompson NJ, Tiang T, et al. Gene expression profiling of esophageal cancer: comparative analysis of Barrett's esophagus, adenocarcinoma, and squamous cell carcinoma. Int J Cancer. 2007;120:1914-21.

5. Bibikova M, Talantov D, Chudin E, Yeakley JM, Chen J, Doucet D, et al. Quantitative gene expression profiling in formalin-fixed, paraffin-embedded tissues using universal bead arrays. Am J Pathol. 2004;165:1799-807.

6. Tusher VG, Tibshirani R, Chu G. Significance analysis of microarrays applied to the ionizing radiation response. Proc Natl Acad Sci U S A. 2001;98: 5116-21.

7. Benjamini Y, Hochberg Y. Controlling the false discovery rate: a practical and powerful approach to multiple testing. J R Stat Soc B. 1995;57:289-300.

8. Bild A, Febbo PG. Application of a priori established gene sets to discover biologically important differential expression in microarray data. Proc Natl Acad Sci U S A. 2005;102:15278-9.

9. Garcia-Escudero R, Paramino JM. Gene expression profiling as a tool for basic analysis and clinical application of human cancer. Mol Carcinog. 2008;47: 573-9.

10. Dahlberg PS, Ferrin LF, Grindle SM, Nelson CM, Hoang CD, Jacobson B. Gene expression profiles in esophageal adenocarcinoma. Ann Thorac Surg. 2004;77: 1008-15.

11. Selaru FM, Zou T, Xu Y, Shustova V, Yin J, Mori Y, et al. Global gene expression profiling in Barrett's esophagus and esophageal cancer: a comparative analysis using cDNA microarrays. Oncogene. 2002;21:475-8.

12. Hao Y, Triadafilopoulos G, Sahbaie P, Yound HS, Omary MB, Lowe AW. Gene expression profiling reveals stromal genes expressed in common between Barrett's esophagus and adenocarcinoma. Gastroenterology. 2006;131:925-33.

13. Kimchi ET, Posner MC, Park JO, Darga TE, Kocherginsky M, Karrison T, et al. Progression of Barrett's metaplasia to adenocarcinoma is associated with the suppression of the transcriptional programs of epidermal differentiation. Cancer Res. 2005;65:3146-54.

14. Guo Y, Chen Z, Zhang L, Zhou F, Shi S, Feng X, et al. Distinctive microRNA profiles relating to patient survival in esophageal squamous cell carcinoma. Cancer Res. 2008;68:26-34.

15. Feber A, Xi L, Luketich JD, Pennathur A, Landreneau RJ, Wu M, et al. MicroRNA expression profiles of esophageal cancer. $J$ Thorac Cardiovasc Surg. 2008;135:255-60.

16. Luthra R, Wu T-T, Luthra MG, Izzo J, Lopez-Alvarez E, Zhang L, et al. Gene expression profiling of localized esophageal carcinomas: association with pathologic response to preoperative chemoradiation. J Clin Oncol. 2006;24: 259-67.

17. Lei W, Liu F, Ness SA. Positive and negative regulation of c-Myb by cyclin D1, cyclin-dependent kinases, and p27 Kip1. Blood. 2005;105:3855-61.

18. Greco C, Alvino S, Buglioni S, Assisi D, Grassi A, Stigliano V, et al. Activation of c-MYC and c-MYB proto-oncogenes is associated with decreased apoptosis in tumor colon progression. Anticancer Res. 2001;21:3185-92.

19. Higashiyama M, Ito T, Tanaka E, Shimada Y. Prognostic significance of osteopontin expression in human gastric carcinoma. Ann Surg Oncol. 2007;14: 3419-27.

20. Yoon H, Liyanarachchi S, Wright FA, Davuluri R, Lockman JC, de la Chapelle A, et al. Gene expression profiling of isogenic cells with different TP53 gene dosage reveals numerous genes that are affected by TP53 dosage and identifies CSPG2 as a direct target of p53. Proc Natl Acad Sci U S A. 2002;99:15632-7.

21. Takaoka M, Kim S-H, Okawa T, Michaylira C, Stairs D, Johnston C, et al. IGFBP-3 regulates esophageal tumor growth through IGF-dependent and independent mechanisms. Cancer Biol Ther. 2007;6:534-40. 